Reprod. Nutr. Dévelop., 1980, 20 (4 B), 1273-1278.

\title{
Le lysozyme dans le matériel et les sécrétions digestives chez le lapin domestique
}

par R. BONNAFOUS, P. RAYNAUD

Laboratoire de Physiologie de la Digestion et de la Nutrition, Instifut de Physiologie,

2, rue François-Magendie, 31400 Toulouse, France

Summary. Lysozyme in the material and the digestive secretions of the domestic rabbit.

Lysozyme is present in the salivary secretions and a low level has been reported for the bile and the pancreatic juice. It has been found to be absent from the digestive material of the rabbit stomach and small and large intestines as well as from the intestinal secretions taken from temporary fistulae placed at the duodenal, jejunal, ileal and colic levels.

However, secretions obtained by permanent intestinal and gastric fisfulae showed high enzyme levels. Lysozyme is thus of probable plasmatic origin. The question of Paneth cell secretion is still to be determined.

\section{Introduction.}

Le lysozyme est une enzyme que l'on rencontre au niveau de nombreux tissus, humeurs et sécrétions digestives. Si sa présence a été depuis longtemps signalée au niveau des glandes salivaires et des larmes, ce n'est que récemment que l'origine du lysozyme intestinal a été démontrée : les cellules de Paneth. Une étude récente récapitulative sur les travaux concernant cette enzyme a été réalisée par Reitamo ef al. (1978).

Chez le lapin, deux observations, en apparence contradictoires, posaient le problème de la production du lysozyme au niveau de la muqueuse intestinale : d'une part, l'absence de l'enzyme au niveau de la muqueuse intestinale pourtant riche en cellules de Paneth, a été rapportée (Catala, 1978) d'autre part, nous avons observé cette même enzyme à des taux relativement élevés dans les sécrétions digestives obtenues par des fistule; chroniques à différents niveaux du tube digestif.

Pour tenter de résoudre ce problème, nous avons recherché la présence de l'enzyme au niveau de différentes humeurs et sécrétions digestives, tout le long de la muqueuse intestinale, du matériel digestif correspondant aux différentes zones de prélèvements de la muqueuse, et dans les sécrétions provenant de fistules, poches aiguës et chroniques. 


\section{Matériel et méthodes.}

Animaux. - Les lapins utilisés pour l'expérience sont des mâles de race commune pesant de 2 à $2,5 \mathrm{~kg}$.

Obtention des sécrétions ef du sang. - Les sécrétions salivaires et les larmes sont obtenues sur des animaux anesthésiés, d̀ l'aide d'une micropipette reliée à un système d'aspiration (trompe à vide).

Les sécrétions pancréatiques et biliaires sont obtenues après fistulisation des canaux sous anesthésie.

Le sang est obtenu sur EDTA au niveau de la veine marginale de l'oreille.

Zones de prélèvements de muqueuse et du matériel. Préparation de la muqueuse. Les broyats de la muqueuse intestinale sont pratiqués à 8 niveaux : estomac (antrum et fundus), intestin grêle (duodénum, jéjunum, iléon), gros intestin (cæcum, côlon proximal antérieur, région du fuseau colique).

La muqueuse est facilement séparée de la musculeuse au niveau de l'estomac et du gros intestin, elle est obtenue après grattage dans l'intestin grêle. Environ $1 \mathrm{~g}$ de muqueuse est dilué au $1 / 10$ (poids/volume) dans du $\mathrm{NaCl}$ à 0,9 p. 100 , passé successivement à l'Ultra-Turrax, $2 \mathrm{~min}$. et au Potter, $1 \mathrm{~min}$ à $0^{\circ} \mathrm{C}$. Les mesures sonf faites à partir du surnageant.

Le matériel est prélevé aux mêmes niveaux et l'activité est mesurée sur le surnageant après centrifugation.

Techniques opératoires.

Anses aiguës. - Les fistules aiguës ont été placées sur l'animal anesthésié aux trois niveaux de l'intestin grêle (duodénum, jéjunum, iléon) et dans la partie médiane du côlon proximal. La portion intestinale choisie est débarrassée de son matériel par pression digitale. Deux ligatures séparées d'environ 8 à $10 \mathrm{~cm}$ obturent le segment intestinal dans lequel est introduit $5 \mathrm{ml}$ de $\mathrm{NaCl}$ à 0,9 p. 100 . L'animal est maintenu anesthésié pendant $3 \mathrm{~h}$. Le liquide présent dans l'anse est alors recueilli et analysé.

Fistules ef poches chroniques. - Des fistules chroniques de Thiry Vella ont été pratiquées au niveau de l'iléon et du côlon proximal. Des poches gastriques chroniques ont été établies au niveau fundique selon la technique décrite par Beauville et Raynaud (1968). Les sucs sont recueillis deux fois par jour pendant la durée de sécrétion.

Techniques analyfiques.

- Le lysozyme (E.C. 3.2.1.17) est dosé selon la technique de Shungar (1952). Les résultałs sont exprimés en $\mu \mathrm{g} / \mathrm{ml}$ accompagnés de l'erreur standard à la moyenne.

- La recherche de fraction active des sucs intestinaux a été réalisée sur Séphadex G. 15, colonne $95 \mathrm{~cm}$; diamètre $1,5 \mathrm{~cm}$. Elution $\mathrm{NaCl}$ à 0,9 p. 100, débit $0,8 \mathrm{ml} / \mathrm{min}$ à 4 o C.

- Les sucs intestinaux sont stockés à $-20^{\circ} \mathrm{C}$ en fonction de leur origine intestinale. Après décongélation, ces sucs sont dyalisés contre l'eau distillée ef lyophilisés. 


\section{Résultats.}

Ils sont consignés dans le tableau 1.

TABLEAU I

Teneur en lysozyme des humeurs, sécrétions digestives, muqueuse et matériel digestif chez le Lapin. Valeurs exprimées en $\mu \mathrm{g} / \mathrm{ml}$ de suc \pm erreur standard à la moyenne

\begin{tabular}{ccccccc}
\hline \multicolumn{7}{c}{ Animaux normaux } \\
\hline $\begin{array}{c}\text { Plasma } \\
(8)\end{array}$ & $\begin{array}{c}\text { Larmes } \\
(6)\end{array}$ & $\begin{array}{c}\text { Salive } \\
(6)\end{array}$ & $\begin{array}{c}\text { Suc } \\
\text { pancréas } \\
(5)\end{array}$ & $\begin{array}{c}\text { Bile } \\
(5)\end{array}$ & $\begin{array}{c}\text { Muqueuse } \\
\text { intestinale } \\
(6)\end{array}$ & $\begin{array}{c}\text { Matériel } \\
\text { digestif } \\
(6)\end{array}$ \\
\hline $10,7 \pm 0,9$ & $52,3 \pm 9,7$ & $3080 \pm 300$ & $4 \pm 1$ & $3,7 \pm 1$ & $/$ & $/\left(^{*}\right)$ \\
\hline
\end{tabular}

\begin{tabular}{ccccc}
\hline \multicolumn{4}{c}{ Animaux fistulés } \\
\hline $\begin{array}{c}\text { Anses } \\
\text { aiguës } \\
(6)\end{array}$ & $\begin{array}{c}\text { Plasma } \\
(8)\end{array}$ & $\begin{array}{c}\text { Fundus } \\
(6)\end{array}$ & $\begin{array}{c}\text { Pléon } \\
(4)\end{array}$ & $\begin{array}{c}\text { Côlon } \\
(4)\end{array}$ \\
\hline$/$ & $46,5 \pm 5$ & $30,7 \pm 4,3$ & $117 \pm 11,5$ & $94 \pm 13$ \\
\hline
\end{tabular}

( ) : nombre d'animaux pour l'expérience.

: activité nulle.

$\left(^{*}\right)$ Exception voir texte.

Au niveau plasmatique, les valeurs relevées chez l'animal normal (10,7 $\pm 0,9 \mathrm{mg} / \mathrm{ml}$ ) sont comparables à celles observées chez l'homme (Dobbins et al., 1976 ; Falchuk, Perroto ef Isselbacher, 1975). La teneur au niveau des larmes est relativement élevée : $52,3 \pm 9,7 \mu \mathrm{g} / \mathrm{ml}$.

Des sécrétions digestives étudiées, c'esł la salive qui présente les valeurs les plus importantes $3080 \pm 300 \mu \mathrm{g} / \mathrm{ml}$. Les sucs pancréatiques et biliaires sont pratiquement dépourvus d'activité respectivement $4 \pm 1$ et $3,7 \pm 1 \mu \mathrm{g} / \mathrm{ml}$.

Nous n'avons jamais observé de lysozyme aux différents niveaux étudiés de la muqueuse intestinale.

Le matériel en provenance des segments intestinaux correspondant aux niveaux de prélèvements de la muqueuse n'a pas présenté d'activité ; une seule exception cependant est à signaler : il concerne les cæcotrophes fraîchement ingérées et récupérées intactes dans la région fundique $(300 \mu \mathrm{g} / \mathrm{ml}$ de surnageant). Nous attribuons cette activité à une forte imprégnation salivaire des cæcotrophes.

Les liquides de perfusion placés dans les anses isolées aiguës ont toujours été dépourvus d'activité.

Les fistules chroniques de type Thiry-Vella et poches isolées gastriques ont permis d'obtenir des sécrétions présentant des teneurs en lysozyme relativement importantes. Les valeurs rapportées représentent les moyennes des données obtenues sur 
des sucs prélevés matin et soir pendant toute la durée des sécrétions (6 à 15 jours). L'activité est décelable dès le lendemain de l'opération, par la suite d'importantes fluctuations du taux de l'enzyme sont observées au cours de la période de sécrétion surtout pour les sucs provenant de l'iléon et du côlon, les valeurs extrêmes étant respectivement $15-210$ ef $10-200 \mu \mathrm{g} / \mathrm{ml}$ de suc (valeurs moyennes respectives $117 \pm 11,5$ et $94 \pm 13 \mu \mathrm{g} / \mathrm{ml}$ ). Les sucs fundiques présentent une teneur en enzyme et des fluctuations plus faibles $12-50 \mu \mathrm{g} / \mathrm{ml}$ (valeur moyenne $30,7 \pm 4,3$ ).

Pendant cette période post-opératoire, les teneurs plasmatiques en lysozyme sont restées anormalement élevées : 46,5 $\pm 5 \mu \mathrm{g} / \mathrm{ml}$.

A ce même niveau, les valeurs extrêmes de 25 à $85 \mu \mathrm{g} / \mathrm{ml}$ rendent compte de fluctuations, certes importantes, mais qui restent cependant nettement plus limitées que celles rapportées pour les sucs iléal ef colique. Une telle observation nous permet de penser que la teneur plasmatique doit se situer à un niveau relativement constant alors que res fluctuations au niveau des sucs intestinaux nous paraissent plus aléatoires.

II ne nous a pas été possible d'établir de corrélation entre les variations relevées au niveau des sécrétions et celles observées au niveau plasmatique.

Enfin, une étude a été réalisée pour rechercher si l'activité mesurée sur les sucs recueillis pouvait être attribuée au lysozyme. Après passage sur Séphadex G. 15 les fractions les plus actives sont éluées à $120 \mathrm{ml}$ (fig. 1). La courbe d'élution du lysozyme standard présente les mêmes caractéristiques. Il s'agit donc d'une molécule de poids moléculaire sensiblement égal au lysozyme.

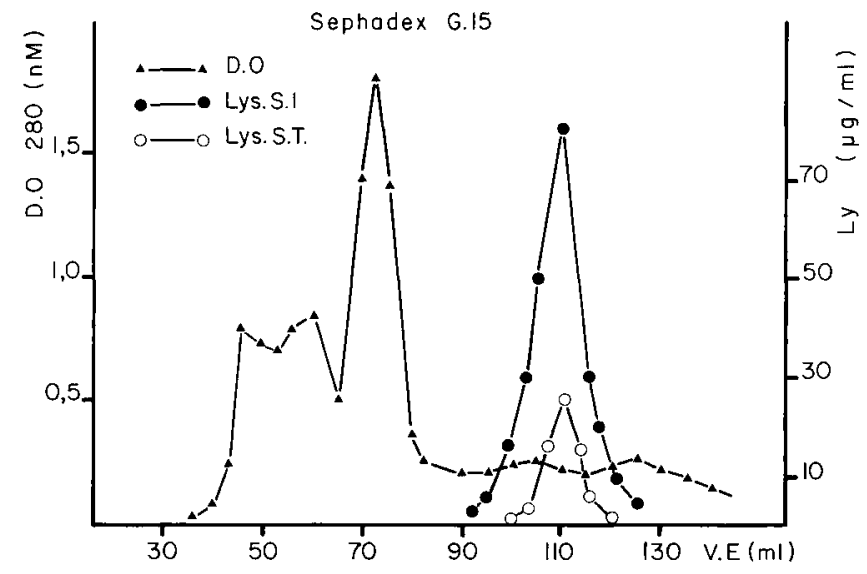

FIG. 1. - Elution de la fraction active en lysozyme à partir du suc intestinal (iléon).

$\mathrm{VE}=$ volume élution $; \mathrm{Ly}=$ lysozyme $;$ Lys. SI ef ST = Lysozyme du suc intestinal et standard.

\section{Discussion.}

De l'étude rapportée par Reitamo ef al. (1978) il ressort que le lysozyme peut être observé au niveau d'un grand nombre de tissus ; mais, c'est au niveau des glandes salivaires et des cellules de Paneth que sa concentration est la plus importante dans le tube digestif. Les cellules de Paneth sont présentes au niveau de l'intestin grêle des oiseaux et de certains mammifères en particulier les Rongeurs (Rat, Souris, Hamster, 
Cobaye, Lapin), les Herbivores (Mouton, Chèvre, Cheval) ; elles ne sont pas représentées chez certains Carnivores (Chien, Chat) (Balas, 1975).

Grâce à l'utilisation d'anticorps, Deckx, Vantrappen ef Parein (1967), Ghoos et Vantrappen (1971), Peeters et Vantrappen $(1974,1975)$ ont pu montrer que les granules des cellules de Paneth contiennent du lysozyme.

Ainsi, pour le Rat (Laval et al., 1978), la Souris (Pradayrol, 1976), le Hamster (Senegas, 1976) et l'Homme (Reitamo et al., 1978) il est actuellement clairement établi que les cellules de Paneth sécrètent le lysozyme.

Chez le Lapin, le lysozyme est présent au niveau des humeurs éfudiées (plasma, larmes). Il est très concentré dans la salive et peu représenté dans les sécrétions pancréatique et biliaire. Il est totalement absent au niveau de la muqueuse intestinale, même dans l'iléon où les cellules de Paneth sont, chez cet animal, bien individualisées et riches en granules de zymogène.

L'absence de l'enzyme au niveau de la muqueuse du matériel intestinal et des sécrétions issues de fistules aiguës et sa présence dans les sécrétions des fistules chroniques accompagnée d'une forte élévation plasmatique de l'enzyme, nous laissent supposer que ce lysozyme a une origine plasmatique ef non intestinale.

En effet, l'enzyme est aussi bien observée dans les sécrétions coliques au niveau intestinal où, à notre connaissance, aucune structure n'est susceptible de la sécréter, que dans les sécrétions iléales où sont situées les cellules de Paneth, réputées pour la sécréter (Laval et al., 1978).

Comment expliquer alors la présence dans les sécrétions en provenance des fistules chroniques? Il est bien connu qu'une élévation plasmatique de lysozyme est toujours observée au cours d'inflammation du tube digestif ef plus particulièrement dans la maladie de Chrohn (Nugent ef al., 1976 ; Klass et Neale, 1978). La confection de fistules chroniques peut être considérée comme responsable de l'élévation plasmatique observée; par la suite, la présence de l'enzyme dans les sécrétions recueillies pourrait s'expliquer soit par le passage de cellules lymphoïdes riches en lysozyme dans la lumière intestinale (Heatley ef Bienenstock, 1979), soit par la libération d'un exsudat riche en enzyme à partir des zones d'inflammation.

Dans de telles conditions, un passage plus ou moins important de cellules lymphoïdes, la libération d'un exsudat variable dans son volume et sa concentration en enzyme dans la lumière intestinale des fistules chroniques, pourraient expliquer les variations aléatoires que nous rapportons.

Cependant, l'absence de lysozyme surtout au niveau de l'iléon, pose le problème de la sécrétion des cellules de Paneth chez cet animal. Nous ne pouvons exclure l'existence de l'enzyme sous une forme inactive (prélysozyme) mais il faudrait alors admettre que la coupure qui s'opère au cours de la synthèse (Palmitter et al., 1977) ne se produit pas chez le Lapin.

Sans faire preuve de finalisme, il est toutefois intéressant de noter que le lysozyme a disparu des sécrétions intestinales de la région intestinale (iléon) précédant l'organe des fermentations bactériennes (cæcum) comme il a disparu des sécrétions salivaires des ruminants (Padgett ef Hirsch, 1967) chez lesquels l'organe des fermentations bactériennes (le rumen) fait suite à la cavité bucco-œsophagienne. 


\section{Références}

BALAS D., 1975. La cellule de Paneth. Efude histologique et histophysiologique. Th. Doct. Etat, Toulouse, $\mathrm{n}^{\circ} 646$.

BEAUVILLE M., RAYNAUD P., 1968. Nouvelle technique de confection d'une poche gastrique isolée chez le lapin. C. R. Acad. Sci., Paris, 267, 1153-1156.

CATALA J., 1978. Recherches sur la physiologie digestive chez le lapin par une étude expérimentale de la fonction pancréatique. Th. Etat, Univ., Paul-Sabatier, Toulouse.

DECKX R. J., VANTRAPPEN G., PAREIN M. M., 1967. Localisation of lysozyme activity in a Paneth cell granule fraction. Biochem. biophys. Acta, 139, 204-207.

DOBBINS J. W.. BINDER H. J., SPIRO H. M., FINCH S. C., 1976. Serum lysozyme in inflammatory bowel disease. Gastroenterology, 70, 469-476.

FALCHUK K. R., PERROTO J. L., ISSELBACHER K. S., 1975. Serum lysozyme in Chrohn's disease. Gastroenterology, 69, 893-896.

GHOOS Y., VANTRAPPEN G., 1971. The cytochemical localisation of lysozyme in Paneth cell granules. Histochem J., 3, 175-178.

HEATLEY R. V., BIENENSTOCK J., 1979. Intestinal lumenal lymphoïd cells. Gastroenterology, 76, 1153.

KLASS H. J., NEALE G., 1978. Serum and fecal lysozyme in inflammatory bowel disease. Gut, 19, 233-239.

LAVAL J., PRADAYROL L., BALAS D., CLEMENTE F., RIBET A., 1978. The quantitative distribution of certain enzymes along the small intestine of the rat and its correlation with the villous area and the Paneth cells. Digestion, 17, 309-316.

NUGENT F., MALLARI R., GEORGE H., RIDLEY N., 1976. Serum lysozyme in inflammaroty bowel disease. Gastroenterology, 70, 1014-1016.

PADGET G. A., HIRSCH J. G., 1967. Lysozyme ; its absence in tears and leucocytes of cattle. J. exp. Biol. med. Sci., 45, 569-570.

PALMITTER R. D., GAGNON J., ERICSSON L. H., WALSH K. A., 1977. Precursor of egg white lysozyme. Amino acid sequence of an $\mathrm{NH}_{2}$ terminal expansion. J. biol. Chem., 252, 6386-6393.

PEETERS T., VANTRAPPEN G., 1974. Study of Paneth cell secretion using lysozyme as marker. Gastroenterology, 66, 758.

PEETERS T., VANTRAPPEN G., 1975. The Paneth cell, a source of intestinal lysozyme. Gut, 16, 553-558.

PRADAYROL L., 1976. La cellule de Paneth. Ełude biochimique du grain sécrétoire. Th. Doct. Spécial., Toulouse, $n^{\circ} 1845$.

REITAMO S., KLOCKARS M., ADINOLFI M., OSSERMAN E. F., 1978. Human lysozyme. La Ricerca, 8, 211-231.

SENEGAS F., 1976. Régulation des mécanismes de sécrétion des cellules de Paneth, entéracytaires et muqueuses du jéjunum de hamster. Th. Doct. Spécial., Toulouse, no 1885.

SHUNGAR D., 1952. Measurement of lysozyme activity and the ultraviolet inactivation of lysozyme. Biochim. biophys. Acto, 8, 302-307. 